\title{
Burnout, Work Satisfaction, and Well-being Among Non-consultant Psychiatrists in Ireland
}

\author{
Caoimhe McLoughlin ${ }^{1} \cdot$ Sarah Casey $^{2} \cdot$ Anna Feeney $^{3} \cdot$ David Weir $^{4} \cdot$ Ahad Ali Abdalla $^{5} \cdot$ Elizabeth Barrett $^{2}$
}

Received: 2 June 2020 / Accepted: 28 October 2020 / Published online: 9 January 2021

(C) Academic Psychiatry 2021

\begin{abstract}
Objective The aim of this study was to explore the areas of psychological well-being, satisfaction at work, and burnout among non-consultant psychiatrists in Ireland, and to assess for potential contributory factors.

Methods The College of Psychiatrists of Ireland distributed the survey online to 100 non-consultant psychiatry doctors working in Ireland. The survey contained questions relating to demographic and work-related variables, the Abbreviated-Maslach Burnout Inventory (a-MBI), Basic Needs Satisfaction at Work (BNSW) scale, and WHO-5 Well-being Index. Descriptive statistics were used by the authors to summarize the data and univariate associations were explored between baseline data and subscales.

Results Sixty-nine percent of our sample completed the survey. Thirty-six percent of the sample met the criteria for burnout, with lack of supervision the only variable significantly associated with this. Lack of regular supervision was associated with lower scores across all work satisfaction domains of the BNSW scale. The WHO-5 Well-being Index identified that 30\% of respondents scored low in personal well-being, indicating that this proportion screened positive for depression, based on international diagnostic criteria. Lack of regular supervision was found to be significantly associated with low psychological well-being.

Conclusion This study indicates that lack of supervision is significantly associated with burnout, lower satisfaction at work, and poorer psychological well-being. Close evaluation of these areas is important to identify vulnerable individuals and areas of training which can be improved upon, which may lead to relevant measures being implemented for the benefit of psychiatrists, patients, and the wider society.
\end{abstract}

Keywords Burnout $\cdot$ Satisfaction $\cdot$ Well-being $\cdot$ Supervision $\cdot$ Psychiatry $\cdot$ Training

The importance of well-being in the workplace is being increasingly recognized, and there is a growing interest in the health of doctors, particularly mental health [1]. In addition to obvious benefits for the individual, clinician well-being is an important indicator of quality patient care [2]. Conversely, poor well-being and work stress can lead to suboptimal deliv-

Caoimhe McLoughlin

cmcloug6@tcd.ie

Tallaght University Hospital, Dublin, Ireland

Temple St University Hospital, Dublin, Ireland

St Patricks University Hospital, Dublin, Ireland

4 Cavan Monaghan Mental Health Services, Monaghan, Ireland

5 University Hospital Limerick Group, Limerick, Ireland ery of patient care, clinician absenteeism, family disruption, depression, and suicide [3].

Burnout is an important mediator of psychological well-being and is now an established occupational health phenomenon [4]. Burnout is characterized by three domains - exhaustion, increased negativism or emotional detachment in relation to one's job, and reduced professional efficacy [4]. It has been shown that psychiatrists tend to suffer from higher levels of psychological distress and burnout relative to other physicians, with female and junior psychiatrists among the most vulnerable $[5,6]$. Increased rates of burnout have been linked with compassion fatigue, vicarious traumatization, and negative transference [7] - factors highly relevant in the day-to-day work of a trainee psychiatrist. A large European study of psychiatry trainees showed the prevalence of severe burnout at $36.7 \%$, with the risk increased by lack of supervision, longer working hours, junior stage of training, and psychiatry not being their first career choice [6]. 
Closely related to burnout and well-being is the phenomenon of work satisfaction - an important influencer of the psychological and physical health of workers [8]. Overall, work satisfaction in psychiatry doctors has generally been reported as high $[9,10]$. There is limited available literature on what influences work satisfaction in psychiatric trainees; however, it has been found that low work satisfaction impacts upon burnout in doctors working in psychiatry [11], and lower levels of satisfaction contribute to attrition in psychiatric training [12].

Poor psychological well-being if not addressed may deteriorate into a defined mental disorder. Studies have found that doctors suffer from considerably higher levels of stress and depression, in addition to higher levels of substance misuse - in particular, alcoholthan the general population [13]. A recent Irish study showed that levels of distress, depression, and anxiety were particularly high in trainees across many specialities [14], and the BoSS study found the prevalence of depression in European psychiatric trainees stood at $20.8 \%$ [15].

Given that emotional difficulties may arise as a consequence of the challenges of the training environment [16, 17], it is imperative that difficulties are addressed early to reduce the negative impact of burnout and depression as one progresses through their career. The training period can be therefore considered a critical time of intervention with regard to the overall well-being and satisfaction of this group. A qualitative study examining the well-being of psychiatry trainees noted that emotional burden and stress often related to the everyday experience of witnessing patients suffering, compounded by feelings of trainee helplessness [16]. Stressful trainee experiences were further exacerbated by trainees feeling "underequipped, overwhelmed, or out of time," and importantly, the authors observed that the level of stress experienced by trainees was compounded by lack of supervision [16]. Worryingly, burnout in psychiatry trainees has also been associated with an increased risk of suicidal ideation [17]. It is essential that training bodies be vigilant to these areas to ensure negative trainee experiences are addressed.

Furthermore, the attrition of trainees that may arise as a result of burnout, poor psychological well-being and low satisfaction only places further strain on resources which, internationally, are often burdened to begin with. Given that the number of consultant psychiatrists in Ireland is less than half the EU average [18], it is crucial that staff retention at trainee level remains optimal to ensure the highest quality of patient care. Thus, the interrelated domains of well-being, burnout and work satisfaction are essential to evaluate and address, as they influence the clinician and patient on an individual level, in addition to impacting the broader healthcare system and wider society.

\section{Methods}

This was a cross-sectional, opt-in online survey, designed using the program SurveyMonkey®. A survey invitation was sent via email link by The College of Psychiatrists of Ireland to a sample of non-consultant hospital doctors (NCHD) working in Psychiatry in Ireland. A non-consultant psychiatry doctor is any doctor working in psychiatry, either in a training or in a non-training post, who is not fully qualified as a consultant. In Ireland, psychiatry training (also known as residency in other countries) is divided into two parts-Basic Specialist Training (BST) and Higher Specialist Training (HST). A trainee must successfully complete both parts before qualifying as a fully independent consultant. A non-training post is a doctor working in psychiatry who is not on a training post or qualified as a consultant. Typically individuals in non-training posts are aiming for placement in a BST or HST post.

This invitation email contained an explanatory statement describing the survey with a link to the questionnaire. We made it clear to the potential participants in the invitation email that their participation was voluntary and that responses would be anonymous and untraceable. Consent was implied by return of the completed survey. Ethics exemption was given by The Royal College of Physicians of Ireland.

For convenience, the sample comprised doctors who were registered to attend the annual NCHD teaching conference ( $n$ $=100$ ). Participants were registered members of The College of Psychiatrists of Ireland and practiced psychiatry in Ireland. Consultants, interns, or doctors who were not practicing psychiatry were not invited to participate. The College of Psychiatrists of Ireland sent the questionnaire directly to participants and collected and anonymized all responses, ensuring privacy of collated data. Two reminder follow-up email links were sent at fortnightly intervals after the initial invite (total of three invitations).

The questionnaire contained demographic and workrelated variables, namely questions about age, gender, years of experience in psychiatry, level of training, place in training post or non-training post, and on-call commitments. Participants were also asked if they received supervision and if psychiatry was their preferred career choice.

\section{Abbreviated-Maslach Burnout Inventory}

The questionnaire contained the Abbreviated-Maslach Burnout Inventory (a-MBI). This is a valid and reliable scale for assessing burnout which correlates well with the longer version of the MBI [19], and has been used extensively in studies assessing burnout in healthcare professionals [20]. The a-MBI is a nine-item scale, with subscales examining three domains: emotional exhaustion (EE), depersonalization (DP), and personal accomplishment (PA). Each subscale is 
assessed by three items. For each item, there is a 7-point Likert scale which ranges from never (0) to every day (6). The score of each subscale ranges from minimum of 0 to a maximum of 18. For emotional exhaustion and depersonalization, higher scores indicated greater levels of burnout, with the inverse applied to personal accomplishment. Validated burnout cutoffs for the a-MBI were used ( $>6$ for depersonalization, $>9$ for emotional exhaustion, and $<9$ for personal achievement) [19]. Consistent with previous studies, respondents were defined as suffering burnout if they scored above the defined cutoff scores in either the depersonalization or emotional exhaustion categories [19].

\section{Basic Needs Satisfaction at Work Scale}

The questionnaire also contained the Basic Needs Satisfaction at Work (BNSW) scale. The BNSW scale examines three domains: "competence," reflecting the need to feel efficient or have work tasks in hand; "autonomy," reflecting the need to experience self-determination at work, and "relatedness," covering the need to have meaningful relations with and feel appreciated by others [21]. These three dimensions measure intrinsic motivations driving people to satisfy basic needs at work, and it is a validated and reliable measure in this context [21, 22]. It consists of 21 items answered on a 7-point Likert scale ranging from 1 ("Not at all true") to 7 ("Very true"). After nine items were reverse scored, the three subscale scores were calculated by averaging item responses. Scores range from 1 to 7 , and the higher the score, the greater the experience of needs satisfaction at work.

\section{WHO-5 Well-being Index}

The questionnaire also contained questions related to psychological well-being, measured by the WHO-5 Well-being Index. The WHO-5 Well-being Index is a short self-reported measure, consisting of five statements which relate to subjective psychological well-being [23]. Respondents rated answers on a Likert scale ranging from 0 ("at no time") to 5 ("all of the time"), according to how they felt over the last 2 weeks. The total raw score $(0-25)$ is multiplied by 4 to give the final score, with higher scores indicating better well-being. The WHO-5 Wellbeing Index has been found to have good validity in measuring for well-being and high sensitivity and specificity in screening for depression $[23,24]$. A WHO- 5 cut-off score of $\leq 50$ is recommended when screening for clinical depression [23].

\section{Analysis}

All analyses were carried out by the authors on IBM Statistical Package for Social Sciences ${ }^{\circledR}$ Version 26. Demographic and work-related data were summarized using descriptive analysis. Continuous data normality was tested using the Shapiro-Wilk test. Likert scales were considered interval data, and internal reliability of the scales was assessed by calculating the Cronbach alpha coefficients for each scale. The association between categorical variables was explored using the chi-square test or Fisher's exact test as appropriate. For continuous variables, the two-sample $t$ test for parametric data and the Wilcoxon ranksum test for non-parametric data were used by the authors. A $p$ value $<0.05$ was used as the level of significance.

\section{Results}

\section{Sample Profile}

Sixty-nine doctors $(69 \%)$ responded to the study. Thirty-two percent were male $(n=22)$, and the median age of respondents was 33 years (IQR 30-40.5). Fifty-four percent of respondents were in a Basic Specialist Training program $(n=37) ; 23 \%$ of respondents were in a Higher Specialist Training program $(n=$ $16)$ and $23 \%$ were in non-training posts $(n=16)$. Eighty-three percent $(n=57)$ received regular supervision, and psychiatry was the preferred career choice for $87 \%(n=60)$. Ninety-three percent of respondents carried out on-call duties with most based in the emergency department $(65 \%, n=45)$ and of at least 24 -h duration $(78 \%, n=54)$. Table 1 summarizes the baseline demographics as well as work-related variables.

\section{Burnout}

The a-MBI was scored from 0 to 18 for each domain. Validated burnout cut-offs were used ( $>6$ for depersonalization, $>9$ for

Table 1 Baseline characteristics

\begin{tabular}{llll}
\hline Age & Years & Median 33 & IQR (30-40) \\
& & $n$ & $\%$ \\
Gender & Male & 47 & $68 \%$ \\
\multirow{3}{*}{ Psychiatry preferred career } & Female & 22 & $32 \%$ \\
& Yes & 60 & $87 \%$ \\
Training level & No & 9 & $13 \%$ \\
& BST & 37 & $54 \%$ \\
Experience in psychiatry & HST & 16 & $23 \%$ \\
& Yocum & 16 & $23 \%$ \\
Regular supervision & & Median 4.5 & IQR $(3-6)$ \\
\multirow{3}{*}{ 24-h on-call } & Yes & $n$ & $\%$ \\
\multirow{2}{*}{ ED on-call } & No & 12 & $83 \%$ \\
& Yes & 54 & $17 \%$ \\
& No & 15 & $78 \%$ \\
& Yes & 45 & $22 \%$ \\
\hline
\end{tabular}


emotional exhaustion, and $<9$ for personal achievement) [19]. The a-MBI scores identified low levels of emotional exhaustion across the sample, with a median score of 6 (IQR 3.5-11) among respondents. Low levels of depersonalization were also identified across the group, with a median score of 3 (IQR 0 5.5). There was a high sense of personal accomplishment among respondents, with a median score of 14 (IQR 12-16.5).

Based on the calculated total score of emotional exhaustion and depersonalization, burnout was present in 25 respondents $(36 \%)$ of the sample. There was adequate internal validity of the subscales based on Cronbach alpha coefficients as presented in Table 2. Of the baseline data presented, only lack of regular supervision was significantly associated with burnout $(p<0.0005)$.

\section{Basic Needs Satisfaction at Work}

The three subscale scores on the Basic Needs Satisfaction at Work (BNSW) scale were calculated by averaging item responses. Scores range from 1 to 7 , and the higher the score, the greater the amount of needs satisfaction being experienced. Results showed that respondents had a moderate sense of autonomy (mean $4.3 \pm 1.1$ ) with a higher sense of competence (mean $5.4 \pm 0.8$ ) and relatedness (mean $5.3 \pm 1$ ). There was high internal validity of the subscales based on Cronbach alpha coefficients as presented in Table 3. The performance of on-call duties in the emergency department was positively associated with higher levels in the autonomy and competence domains. Lack of regular supervision was associated with lower scores across all three subdomains of the BNSW scale-see Table 4.

\section{Psychological Well-being}

The WHO-5 Well-being Index identified 21 respondents (30\%) who scored low in personal well-being. A score of less than 50 indicates screening positive for depression, based on international diagnostic criteria $[23,24]$. On univariate analysis, only lack of regular supervision was found to be a significant predictor of low personal well-being $(p<0.05)$. There
Table 3 Basic Needs Satisfaction at Work subscale scores

\begin{tabular}{llll}
\hline Subscale & Mean & SD & Cronbach's alpha \\
\hline Autonomy & 4.4 & 1.12 & 0.839 \\
Competence & 5.41 & 0.84 & 0.703 \\
Relatedness & 5.31 & 0.98 & 0.847 \\
\hline
\end{tabular}

was high internal validity of the scale as evidenced by a Cronbach alpha coefficient of 0.918 .

\section{Discussion}

While our sample indicated low levels of emotional exhaustion and depersonalization and high levels of personal accomplishment in the burnout domains, the prevalence of burnout at $36 \%$ remains concerning. Our study broadly reflects the norms reported in this population in the existing literature with a recent systematic review showing the overall prevalence of burnout in psychiatry trainees at $33.6 \%$ [25]. The demonstrated association between inadequate supervision and burnout in this study is in keeping with previous studies [6, 25]. Maslach and Leiter outline the unique pressures for psychiatrists that may predispose to burnout - dealing with the effects of trauma, becoming the target of anger or paranoia, or the risk of violence towards the clinician or others [7]. The chronic nature of some psychiatric disorders means recovery can be slow and treatment options limited - such factors are likely to exacerbate feelings of incompetence and stress at trainee level.

Regarding work satisfaction, our results showed that the majority of respondents were reasonably satisfied, again comparable with findings from other countries, [9, 10]. The performance of ED duties was positively associated with higher levels in the autonomy and competence domains of the BNSW scale. This finding is perhaps unsurprising given that ED work is diverse and typically requires plenty of proactive problem solving - inherently incorporating high skill variety, task identity, and task significance-domains which have

Table 2 a-MBI subscale scores

\begin{tabular}{llllll}
\hline Subscale & Median (IQR) & Category & Frequency & Percent & Cronbach's alpha \\
\hline Personal achievement & \multirow{2}{*}{$14(12-16.5)$} & Burnout & 4 & $6 \%$ & 0.603 \\
& & No burnout & 65 & $94 \%$ & \\
Emotional exhaustion & $6(3.5-11)$ & Burnout & 23 & $33 \%$ & 0.858 \\
& & No burnout & 46 & $67 \%$ & \\
Depersonalization & $3(0-5.5)$ & Burnout & 6 & $9 \%$ & 0.652 \\
Overall burnout & \multirow{2}{*}{$9(5-15)$} & No burnout & 63 & $91 \%$ & \\
& & Burnout & 25 & $36 \%$ & 0.777 \\
\hline
\end{tabular}


Table 4 Basic Needs Satisfaction at Work subscale scores and association with supervision

\begin{tabular}{llllll}
\hline Subscale & Regular supervision & $N$ & Mean score & SD & $p$ value for difference \\
\hline Autonomy & Yes & 57 & 4.56 & 1.05 & \\
& No & 12 & 3.64 & 1.21 & $<0.01$ \\
Competence & Yes & 57 & 5.51 & 0.80 & \\
& No & 12 & 4.94 & 0.86 & $<0.05$ \\
Relatedness & Yes & 57 & 5.48 & 0.87 & $<0.005$ \\
& No & 12 & 4.50 & 1.08 & $<$ \\
\hline
\end{tabular}

been found to positively impact motivation and satisfaction at work [26]. While this is a positive finding in relation to work satisfaction in the ED setting, it must be viewed cautiously with respect to the additional stressors associated with the nature of this work - as frontline workers typically experience high burnout, especially ED clinicians [27].

Our results indicate $30 \%(n=21)$ of respondents scored low on subjective psychological well-being, indicating a positive screening result for depression [24]. There are many factors that may contribute to poor psychological well-being in this group — such as exposure to patient suicide and the interpersonal burden inherent in the work itself. In a recent large Irish survey of doctors using the WHO-5 well-being index, $46 \%$ of doctors in psychiatry including consultants reported low subjective well-being [14]. The reason for a lower prevalence of poor psychological well-being in our sample could be related to a responder bias, and our sample did not include consultants. It is notable that a large systematic review and meta-analysis estimated the prevalence of depressive symptoms at $28.8 \%$ among resident physicians, comparable with our figures [28].

There is some evidence that satisfaction, burnout, and psychological well-being are related, though we did not evaluate the relationship in this study. The relationship between work satisfaction and burnout was examined in a large New Zealand study of psychiatry doctors, with domains related to lower satisfaction found to be significantly associated with high emotional exhaustion [9]. In a large survey of Canadian physicians, $53 \%$ of residents scored low on psychological well-being as measured by the WHO-5 Well-being Index, with personal burnout and work dissatisfaction significantly predicting WHO-5 well-being scores [29]. It has also been proposed that physician turnover may be related to job satisfaction partly via the mediating effect of occupational burnout [30], which suggests that addressing these areas will reduce staff attrition and intent to leave.

Seventeen percent of respondents in our study $(n=12)$ did not receive regular supervision, slightly higher than that reported in a previous European study [6]. The characteristics of respondents across the sample who did not receive supervision were not significantly different from those who did; however, there may be unmeasured factors impacting on this. Though broadly defined in the literature, the primary goals of supervision are to support professional development and ensure patient safety [31]. Psychiatry is arguably distinct from other areas of medicine insofar as it combines aspects of medicine with aspects of psychotherapy, ascribing supervision a somewhat unique role in training. Our study showed that lack of supervision was shown to be significantly associated with higher burnout scores, lower work satisfaction, and poorer psychological well-being in this group, suggesting that paying due regard to supervision may have a positive impact on these areas.

There have been limited empirical studies examining the effectiveness of supervision practices in psychiatry, and guidelines on recommended supervision practices have not been clearly defined $[31,32]$. Part of the problem is the lack of development of validated scales to assess the effectiveness of supervision [33]. McDonald and Ellis describe the urgent need for more evidence-based research on the area of supervision in psychiatry - describing it as a "terra incognita" [32]. The supervisee/supervisor relationship has been described as one of the most important aspects to supervision in clinical practice generally [31].

Though under-researched in psychiatric trainee settings, the positive effect of supervision on clinician well-being and burnout has been demonstrated elsewhere, including community mental health settings [34, 35]. The Manchester Clinical Supervision Scale (MCSS) has been used to evaluate supervision mainly in nursing settings and looks at seven areas"trust and rapport," "advice/support," "improved care/skills," "supervision value," "finding time," "personal issue," and "reflection"[33]. Using this model, a large multi-center study of Finnish nurses and another study of community mental health nurses found that supervisees' evaluations of "trust and rapport," "advice and support," and "finding time for supervision" predicted burnout and job satisfaction $[35,36]$. A large UK study of trainee doctors examined the extent to which the trainee felt clinically supported during their training - evaluating specific domains such as accessibility of supervisors and having to cope with problems beyond their competence. When approached effectively, these domains, along with the delivery of appropriate feedback, personal progress meetings, and regular performance assessment, were found to increase trainee satisfaction in this study [37]. These 
studies further emphasize the importance of the supervision relationship in reducing burnout and work dissatisfaction.

This study provides new information on the relationship between supervision and well-being and satisfaction at work in non-consultant psychiatry doctors. This study is relevant given the importance of continual striving for excellent training standards and recruitment and retention difficulties for doctors in both Ireland and abroad. The study used validated instruments in the measure of burnout, satisfaction at work, and well-being, allowing for comparison with other studies. There was a high response rate from those invited to complete the survey. The high uptake possibly reflects an enthusiastic sample who attended the NCHD teaching conference and were interested in this topic; however, therein lies a risk of responder bias. The survey was cross-sectional and a selfreport measure which means there is a risk of recall bias, and one cannot determine whether the associations observed are causally related. We had limited available data on other factors that influence these domains such as personal demographic variables and mental health parameters, which limited analysis to an extent; however, this was an important measure to protect the anonymity of responders.

Going forward to address these issues, there is increasing recognition that the implementation of groups such as Schwartz rounds impacts on staff satisfaction [38]; and the provision of Balint groups has also shown some promise in reducing physician burnout [39]. The important role of supervision has been highlighted in this study and elsewhere, and though busy services may perhaps impact on supervisor and trainee availability, this is likely the time when supervision is needed most, and it is important that supervisors are supported in this vein. The CAP-STATE study [40] recognizes the need for interventions to support training institutions - -highlighting the importance of allocating dedicated time for trainers to support and teach trainees, so that they in turn may become competent trainers themselves.

In conclusion, this study has important implications for psychiatric training which arguably extend to other medical specialities. Those not receiving supervision report poorer psychological well-being, lower satisfaction at work, and higher levels of burnout. Since the completion of our study, our healthcare landscape has fundamentally changed with the advent of COVID-19. The long-term impact of the pandemic on our healthcare systems is impossible to estimate-however it is certain that the global crisis has highlighted the emotional impact of the work of psychiatrists, and represents an opportunity to critically address these areas, and develop robust, effective supports for this vulnerable group.

Acknowledgments The authors would like to acknowledge the respondents who took the time to complete the survey.

\section{Compliance with Ethical Standards}

Disclosures On behalf of all authors, the corresponding author states that there is no conflict of interest.

\section{References}

1. Peters M, Hasan O, Puddester D, Garelick A, Holliday C, Rapanakis T, et al. Doctors' health: taking the lifecycle approach. BMJ. 2013;347:f7086.

2. Wallace JE, Lemaire JB, Ghali WA. Physician wellness: a missing quality indicator. Lancet. 2009;374:1714-21. https://doi.org/10. 1016/S0140-6736(09)61424-0.

3. Bhui K, Dinos S, Stansfeld S, White P. A synthesis of the evidence for managing stress at work: a review of the reviews reporting on anxiety, depression, and absenteeism. J Environ Public Health. 2012;2012:1-21.

4. World Health Organization Burn-out an "occupational phenomenon": International Classification of Diseases, 2019. Last accessed: 04/23/2020 Available: https:/www.who.int/mental_health/ evidence/burn-out/en/.

5. Senthil F. Personal resilience for psychiatrists: systematic review. BJPsych Bull. 2019;43(6):295.

6. Jovanović N, Podlesek A, Volpe U, Barrett E, Ferrari S, Rojnic Kuzman M, et al. Burnout syndrome among psychiatric trainees in 22 countries: risk increased by long working hours, lack of supervision, and psychiatry not being first career choice. Eur Psychiatry. 2016;32:34-41

7. Maslach C, Leiter MP. Understanding the burnout experience: recent research and its implications for psychiatry. World Psychiatry. 2016;15:103-11. https://doi.org/10.1002/wps.20311.

8. Faragher AEB, Cass M, Cooper CL, Faragher EB, Cass M, Cooper $\mathrm{CL}$, et al. The relationship between job satisfaction and health: a meta-analysis. Occup Environ Med. 2005;62:105-12. https://doi. org/10.1136/oem.2002.006734.

9. Kumar S, Fischer J, Robinson E, Hatcher S, Bhagat R. Burnout and job satisfaction in New Zealand psychiatrists: a national study. Int J Soc Psychiatry. 2007;53(4):306-16.

10. Prosser D, Johnson S, Kuipers E, Szmukler G, Bebbington P, Thornicroft G. Mental Health, 'burnout' and job satisfaction among hospital and community-based mental health staff. Br J Psychiatry. 1996;169(3):334-7.

11. Kumar S, Sinha P, Dutu G. Being satisfied at work does affect burnout among psychiatrists: a national follow-up study from New Zealand. Int J Soc Psychiatry. 2012;59(5):460-7.

12. Lau T, Kumar S, Lau T, Kumar S, Robinson E. New Zealand's psychiatrist workforce: profile, recruitment and retention. Aust N Z J Psychiatry. 2004;38(7):547-53.

13. Firth-Cozens J. Improving the health of psychiatrists. Adv Psychiatr Treat. 2007;13(3):161-8.

14. Hayes B, Prihodova L, Walsh G, Doyle F, Doherty S. What's up doc? A national cross-sectional study of psychological wellbeing of hospital doctors in Ireland. BMJ Open. 2017;7(10):e018023.

15. Beezhold J, Durant D, Ginzler A, Sherman R, Podlesek A, Jovanovic N. Prevalence of depression in psychiatry trainees in 22 countries: findings from the international burnout syndrome study (BoSS). Eur Psychiatry. 2017;41:S569.

16. Benson N, Chaukos D, Vestal H, Chad-Friedman E, Denninger J, Borba C. A qualitative analysis of stress and relaxation themes contributing to burnout in first-year psychiatry and medicine residents. Acad Psychiatry. 2018;42(5):630-5.

17. Tateno M, Jovanović N, Beezhold J, Uehara-Aoyama K, UmeneNakano W, Nakamae T, et al. Suicidal ideation and burnout among 
psychiatric trainees in Japan. Early Interv Psychiatry. 2017;12(5): 935-7.

18. Health resources - Doctors - OECD Data [Internet]. theOECD. 2020 [Accessed 12 May 2020]. Available from: https://data.oecd. org/healthres/doctors.htm.

19. Riley M, Mohr D, Waddimba A. The reliability and validity of three-item screening measures for burnout: evidence from groupemployed health care practitioners in upstate New York. Stress Health. 2017;34(1):187-93.

20. Rotenstein L, Torre M, Ramos M, Rosales R, Guille C, Sen S, et al. Prevalence of burnout among physicians. JAMA. 2018;320(11): 1131-50.

21. Deci E, Ryan R, Gagné M, Leone D, Usunov J, Kornazheva B. Need satisfaction, motivation, and well-being in the work organizations of a former Eastern Bloc country: a cross-cultural study of self-determination. Personal Soc Psychol Bull. 2001;27(8):930-42.

22. Colledani D, Capozza D, Falvo R, Di Bernardo GA. The workrelated basic need satisfaction scale: an Italian validation. Front Psychol. 2018;9:1859.

23. Topp C, Østergaard S, Søndergaard S, Bech P. The WHO-5 Wellbeing Index: a systematic review of the literature. Psychother Psychosom. 2015;84(3):167-76.

24. Krieger T, Zimmermann J, Huffziger S, Ubl B, Diener C, Kuehner $\mathrm{C}$, et al. Measuring depression with a well-being index: further evidence for the validity of the WHO Well-Being Index (WHO-5) as a measure of the severity of depression. J Affect Disord. $2014 ; 156: 240-4$.

25. Chan M, Chew Q, Sim K. Burnout and associated factors in psychiatry residents: a systematic review. Int J Med Educ. 2019;10: 149-60.

26. Oldham GR, Hackman JR. How job characteristics theory happened. In: Smith KG, Hitt MA, editors. The Oxford handbook of management theory: the process of theory development. Oxford: Oxford University Press; 2005. p. 151-70.

27. Arora M, Asha S, Chinnappa J, Diwan A. Review article: burnout in emergency medicine physicians. Emerg Med Australas. 2013;25(6):491-5.

28. Mata D, Ramos M, Bansal N, Khan R, Guille C, Di Angelantonio $\mathrm{E}$, et al. Prevalence of depression and depressive symptoms among resident physicians a systematic review and metanalysis. JAMA. 2015;314(22):2373.
29. Kassam A, Horton J, Shoimer I, Patten S. Predictors of well-being in resident physicians: a descriptive and psychometric study. J Grad Med Educ. 2015;7(1):70-4.

30. Zhang Y, Feng X. The relationship between job satisfaction, burnout, and turnover intention among physicians from urban stateowned medical institutions in Hubei, China: a cross-sectional study. BMC Health Serv Res. 2011;11:235.

31. Kilminster S, Jolly B. Effective supervision in clinical practice settings: a literature review. Med Educ. 2000;34(10):827-40.

32. MacDonald J, Ellis P. Supervision in psychiatry: terra incognita? Curr Opin Psychiatry. 2012;25(4):322-6.

33. Winstanley J. Manchester clinical supervision scale. Nurs Stand. 2000;14(19):31-2.

34. Koivu A, Saarinen P, Hyrkas K. Who benefits from clinical supervision and how? The association between clinical supervision and the work-related well-being of female hospital nurses. J Clin Nurs. 2012;21(17-18):2567-78.

35. Edwards D, Burnard P, Hannigan B, Cooper L, Adams J, Juggessur $\mathrm{T}$, et al. Clinical supervision and burnout: the influence of clinical supervision for community mental health nurses. J Clin Nurs. 2006;15(8):1007-15.

36. Hyrkäs K, Appelqvist-Schmidlechner K, Haataja R. Efficacy of clinical supervision: influence on job satisfaction, burnout and quality of care. J Adv Nurs. 2006;55(4):521-35.

37. Gregory S, Demartini C. Satisfaction of doctors with their training: evidence from UK. BMC Health Serv Res. 2017;17(1):851.

38. Taylor C, Xyrichis A, Leamy MC, et al. Can Schwartz center rounds support healthcare staff with emotional challenges at work, and how do they compare with other interventions aimed at providing similar support? A systematic review and scoping reviews. BMJ Open. 2018;8:e024254. https://doi.org/10.1136/bmjopen2018-02425.

39. West C, Dyrbye L, Erwin P, Shanafelt T. Interventions to prevent and reduce physician burnout. Obstet Gynecol Surv. 2017;72(3): 147-9.

40. Barrett E, Jacobs B, Klasen J. The child and adolescent psychiatry: study of training in Europe (CAP-STATE). Eur Child Adolesc Psychiatry. 2020;29:11-27.

Publisher's Note Springer Nature remains neutral with regard to jurisdictional claims in published maps and institutional affiliations. 